\title{
A randomised, placebo controlled clinical trial of the aldose reductase inhibitor CT-112 as management of corneal epithelial disorders in diabetic patients
}

\author{
M Nakahara, K Miyata, S Otani, T Miyai, R Nejima, S Yamagami, S Amano
}

Br J Ophthalmol 2005;89:266-268. doi: 10.1136/bjo.2004.049841

Aim: To evaluate the efficacy of topical aldose reductase inhibitor CT-112 (5-[3-ethoxy-4-pentyloxyphenyl]-2,4-thiazolidinedione) on corneal epithelial barrier function in diabetic patients.

Methods: This was a prospective, randomised, double masked placebo controlled study. 34 eyes of 34 diabetic patients were randomly assigned treatment with $0.25 \%$ eye drops of CT-112 $(n=22)$ or a placebo $(n=12)$ four times a day for 8 weeks. Corneal fluorescein staining and corneal sensation were examined before treatment as well as 4 and 8 weeks after administration. Corneal epithelial permeability to fluorescence was measured with an anterior fluorophotometer.

Results: Average scores of superficial punctate keratopathy and corneal sensitivity did not differ significantly between the two groups at any time. Whereas average fluorescein concentrations did not differ significantly for the CT-112 and placebo groups before treatment, they did differ significantly 4 and 8 weeks after treatment (4 weeks, $p=0.0327 ; 8$ weeks, $p=0.0143$ ).

Conclusion: The topical aldose reductase inhibitor, CT-112 improves the corneal epithelial barrier function in diabetic patients.

D iabetic patients are vulnerable to such corneal epithelial disorders as superficial punctate keratopathy (SPK) and epithelial erosion. ${ }^{1}$ Moreover, damage to the corneal epithelium during vitreous surgery and retinal photocoagulation sometimes induces vision threatening corneal epithelial complications such as persistent epithelial defects and recurrent erosion. ${ }^{2-5}$ Two mechanisms have been proposed for these diabetic keratopathies, an activated polyol pathway $^{6-8}$ and accumulation of advanced glycation end products (AGE) in the basement membrane of the corneal epithelium. ${ }^{9}$ Efficacies of inhibitors of aldose reductase, the first enzyme of polyol pathway, have been evaluated as antipolyol pathway drugs. The oral aldose reductase inhibitor (ARI) ONO-2235 decreases corneal epithelial changes in diabetic patients through recovery of corneal sensation and tear production..$^{11}$ In addition, topical ARI, CT-112 (5-[3ethoxy-4-pentyloxyphenyl]-2,4-thiazolidinedione) improves corneal sensation in clinical cases ${ }^{12}$ and corneal epithelial barrier function in galactose fed rats. ${ }^{13}$ These findings are suggestive but not conclusive. We therefore established a randomised, double masked clinical trial in which diabetic patients were treated topically with CT-112 or a placebo; we evaluated changes in corneal fluorescein staining based on our earlier reported scoring method ${ }^{14}$ and measured corneal epithelial permeability to fluorescence with an anterior fluorophotometer.

\section{PATIENTS AND METHODS}

This was a prospective, randomised, double masked, placebo controlled study. The research protocol adhered to the tenets of the Declaration of Helsinki. Participants comprised a consecutive series of diabetic patients who visited Miyata Eye Hospital. Exclusion criteria were contact lens wearer, tear deficiency (less than $5 \mathrm{~mm}$ in Schirmer test), active ocular infection, atopic keratoconjunctivitis, and severe superficial punctate keratopathy graded more than AlDl in our earlier reported method. ${ }^{14}$ In addition, other exclusion criteria were the intake of oral ARI within 6 months and the use within 30 days of ocular ointments and eye drops such as sodium hyaluronate, sodium chondroitin sulfate, vitamin $\mathrm{B}_{2}$, and other drugs known to modify the ocular surface. After we obtained their informed consent, 36 diabetic patients were enrolled and randomly assigned to treatment with $0.25 \%$ eye drops of CT-112 (Senju Pharmaceutical, Osaka, Japan) or a placebo four times a day for 8 weeks. The placebo was the base solution of CT-112. Two patients in the CT-112 group were excluded because one was found to have used a prohibited eye drop before treatment and the other could not undergo the 8 week follow up examination. In total, 34 patients (22 in the CT-112 treated group and 12 in the placebo group) were followed for the duration of the study.

SPK magnitude was scored according to our reported method $^{14}$ that uses lesion area and density as parameters. Scores are defined in table 1 . Area grade is defined as the total SPK lesion area. Grading is shown as combined of area and density grades-for example, A2D3. In this study, the sum of the area and density grades has been designated the score for each eye.

Corneal epithelial permeability to fluorescence was measured with a slit lamp fluorophotometer (FL-500, Kowa, Tokyo) as described previously. ${ }^{14}{ }^{15}$ Briefly, autofluorescence intensity was measured at the central corneal stroma before the instillation of fluorescein, and the value obtained designated the background fluorescence. Sodium fluorescein ( $3 \mu \mathrm{l}$; Alcon, Fort Worth, TX, USA) distilled to $0.5 \%$ in balanced salt solution (BSS Plus, Alcon) was instilled to the lower conjunctival sac through a micropipette. Ten minutes after instillation, the ocular surface was washed thoroughly with $20 \mathrm{ml}$ of BSS PLUS. Thirty minutes after instillation, fluorescence intensity was measured at the central corneal stroma. The background intensity was subtracted from the average of 10 measurements. Values obtained were converted to fluorescein concentrations based on calibration curves obtained with a $0.5 \mathrm{~mm}$ wide slide glass corneal model. ${ }^{16}{ }^{17}$

Corneal sensitivity was measured with a Cochet-Bonnet anaesthesiometer. Nylon length measured in millimetres was

Abbreviations: AGE, advanced glycation end products; ARI, aldose reductase inhibitor; BSS, balanced salt solution; SPK, superficial punctate keratopathy 
Table 1 Definitions of the area and density grades of superficial punctate keratopathy

$\begin{array}{ll}\text { Area (total sum of the affected area) } \\ \text { A0: } & \text { no punctate staining } \\ \text { A1: } & \text { the area occupies less than one third of the cornea } \\ \text { A2: } & \text { the area occupies one third to two thirds of the cornea } \\ \text { A3: } & \text { the area occupies more than two thirds of the cornea } \\ \text { Density } & \\ \text { D0: } & \text { no punctate staining } \\ \text { D1: } & \text { sparse density } \\ \text { D2: } & \text { moderate density } \\ \text { D3: } & \end{array}$

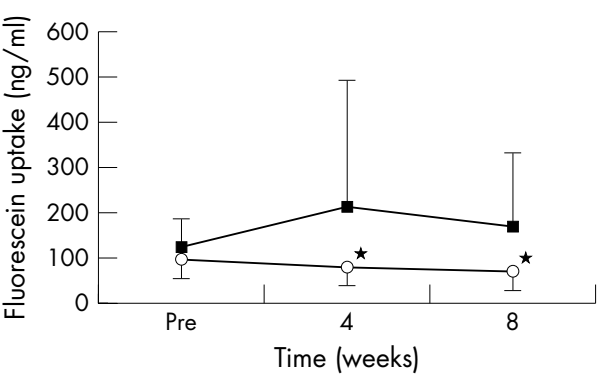

Figure 1 Time courses of average fluorescein concentrations and standard deviations before and after treatment of the CT- 112 lopen circles) and control groups (solid squares). The average fluorescein concentrations do not differ significantly between the groups before treatment but do differ significantly 4 and 8 weeks after treatment ( 4 weeks, $p=0.0327 ; 8$ weeks, $p=0.0143$ ).

converted to mean pressure values $\left(\mathrm{g} / \mathrm{mm}^{2}\right)$ by means of the conversion table provided by the manufacturer.

The unpaired $t$ test or Mann-Whitney $\mathrm{U}$ test was used to compare preoperative background data and pretreatment and post-treatment examination data for the CT-112 and placebo groups. A p value of less than 0.05 was considered significant.

\section{RESULTS}

Background information on the 34 patients is given in table 2 . All had type 2 diabetes mellitus. None of the parameters differed significantly between the two groups.

Average SPK scores for the CT-112 and placebo groups before treatment and 4 and 8 weeks after are shown in table 3. These scores did not differ significantly between the two groups at any time point.
Average corneal sensitivities in the CT-112 and placebo groups before treatment and 4 and 8 weeks after are shown in table 4. Average corneal sensitivity did not differ significantly between the two groups at any time point.

Time courses of the average fluorescein concentrations in the CT-112 and control groups before and after treatment are shown in figure 1. Although the average concentrations did not differ significantly before treatment, they did differ significantly 4 and 8 weeks after it $(4$ weeks, $p=0.0327$; 8 weeks, $p=0.0143$ ).

\section{DISCUSSION}

The anterior fluorophotometer used detects subclinical changes in the corneal epithelium by quantitatively measuring its barrier function. ${ }^{16-20}$ In Göbbels' and our previous studies, ${ }^{15} 21$ when the fluorophotometer was used, the average fluorescein concentrations in the diabetic patients were significantly higher than in the non-diabetic controls, indicative that the corneal epithelial barrier function is impaired in diabetic patients. This study showed that average fluorescein uptakes in the CT-112 group after 4 and 8 weeks of treatment were significantly less than in the placebo group, whereas there was no significant difference between the groups before treatment, evidence that there was recovery from impairment of corneal epithelial barrier function in the diabetic patients after topical application of CT-112.

In diabetic patients, increased epithelial permeability to fluorescein is correlated positively with the duration of diabetes mellitus and $\mathrm{HbA}_{\mathrm{Ic}}{ }^{15}{ }^{21}$ In our study, no significant differences were found in the background data for the CT-112 and control groups, confirmation of the validity of the comparison between the two groups. On the other hand, the study subjects were heterogeneous in terms of duration of diabetes mellitus, absence/presence of retinopathy, and preoperative ocular surface status. A future study with larger population is needed to clarify the effects of these background status.

ARI is posited to improve the corneal epithelial changes caused by diabetes through the recovery of corneal sensation and increased tear production. ${ }^{10}{ }^{11}$ In our study, however, the application of CT-112 improved corneal epithelial barrier function but did not change corneal sensation or the fluorescein staining score. With the method we used, it may have been difficult to detect any subtle improvement in corneal sensation and change in the fluorescein score because our diabetic participants happened to have low fluorescein staining scores and almost normal corneal sensation, even before treatment. In contrast, change in corneal barrier

Table 2 Participants' characteristics

\begin{tabular}{llll}
\hline & CT-112 group & Placebo group & p Value \\
\hline No of participants & 22 & 12 & \\
Age (years) & $69.5(9.85)$ & $69.4(7.63)$ & $0.15^{*}$ \\
Duration of diabetes mellitus (years) & $18.2(8.88)$ & $19.7(8.93)$ & $0.67^{*}$ \\
$\mathrm{HbA}_{1 \mathrm{c}}(\%)$ & $7.97(1.44)$ & $7.52(1.28)$ & $0.37^{*}$ \\
Schirmer test $(\mathrm{mm} / 5 \mathrm{~min})$ & $9.8(3.5)$ & $12.0(6.4)$ & $0.21^{*}$ \\
\hline *Unpaired $t$ test. & & & \\
\hline
\end{tabular}

Table 3 Time course changes in superficial punctate keratopathy scores

\begin{tabular}{llll}
\hline & CT-112 group & Placebo group & p Value \\
\hline Pretreatment & $0.46(0.86)$ & $0.67(0.99)$ & $0.52^{*}$ \\
4 weeks & $0.82(1.18)$ & $0.50(0.91)$ & $0.42^{*}$ \\
8 weeks & $0.82(1.18)$ & $0.67(0.99)$ & $0.71^{*}$ \\
\hline *Mann-Whitney U test. & & & \\
\hline
\end{tabular}


Table 4 Time course changes in corneal sensitivity

\begin{tabular}{llll}
\hline & CT-1 12 group $\left(\mathbf{g} / \mathrm{mm}^{2}\right)$ & Placebo group $\left(\mathbf{g} / \mathrm{mm}^{2}\right)$ & $\mathrm{p}$ Value \\
\hline Pretreatment & $1.00(0.19)$ & $0.99(0.08)$ & $0.91^{*}$ \\
4 weeks & $0.96(0)$ & $0.96(0)$ & $0.99^{*}$ \\
8 weeks & $0.97(0.04)$ & $0.97(0.04)$ & $0.94^{*}$ \\
\hline *Mann-Whitney U test. & & &
\end{tabular}

function can be detected with an anterior fluorophotometer capable of detecting subtle subclinical changes.

Göbbels' and our previous studies ${ }^{15} 21$ showed that the average fluorescein uptake was significantly higher in the diabetic patients than in the non-diabetic controls, indicative that corneal epithelial barrier function is weakened in diabetic patients. Once this function is weakened, the cornea may become more vulnerable to such foreign pathogens as bacteria and fungi. One study showed a $12 \%$ incidence of diabetes mellitus in a group of patients with fungal keratitis although the incidence of diabetes mellitus in the US population is estimated to be only $1 \%{ }^{22}$ Impaired corneal epithelial barrier function probably is one reason for the predisposition of diabetic patients for corneal infection.

Our earlier report suggested that the accumulation of AGE on the basement membrane of the corneal epithelium has a causative role in corneal epithelial disorders found in diabetic patients. ${ }^{9}$ In that study, AGE accumulated on the epithelial basement membrane in corneas of diabetic patients, and non-enzymatic glycation of laminin on culture dishes attenuated the adhesion and spread of corneal epithelial cells in vitro. Previous studies ${ }^{23-25}$ reported that fructose, a polyol pathway metabolite, enhances non-enzymatic glycation reaction, therefore aldose reductase inhibitor may improve corneal epithelial barrier function by attenuating AGE formation in the cornea. This hypothesis needs to be tested by further experiments.

\section{Authors' affiliations \\ M Nakahara, K Miyata, S Otani, T Miyai, R Nejima, Meiwakai Medical Foundation, Miyata Eye Hospital, Miyakonojo, Miyazaki, Japan S Yamagami, S Amano, Department of Ophthalmology, University of Tokyo School of Medicine, Tokyo, Japan \\ The authors have no commercial or proprietary interest in the products or companies mentioned in the current article.}

Correspondence to: Shiro Amano, 7-3-1 Hongo, Bunkyo-ku, Tokyo, 113-8655 Japan; amanos-tky@umin.ac.jp

Accepted for publication 1 August 2004

\section{REFERENCES}

1 Schultz RO, Van Horn DL, Peters MA, et al. Diabetic keratopathy. Trans Am Ophthalmol Soc 1981;79:180-9.

2 Perry HD, Foulks GN, Thoft RA, et al. Corneal complications after closed vitrectomy through the pars plana. Arch Ophthalmol 1978;96:1401-3.

3 Brightbill FS, Myers FL, Bresnick GH. Postvitrectomy keratopathy. Am J Ophthalmol 1978;85:651-5.
4 Foulks GN, Thoft RA, Perry HD, et al. Factors related to corneal epithelial complications after closed vitrectomy in diabetics. Arch Ophthalmol 1979;97:1076-8.

5 Chung $\mathrm{H}$, Tolentino $\mathrm{Fl}$, Cajita VN, et al. Reevaluation of corneal complications after closed vitrectomy. Arch Ophthalmol 1988;106:916-9.

6 Kinoshita JH, Fukushi S, Kador P, et al. Aldose reductase in diabetic complications of the eye. Metabolism 1979;28:462-9.

7 Cogan DG, Kinoshita JH, Kador PF, et al. Aldose reductase and complications of diabetes. Ann Intern Med 1984;101:82-91.

8 Akagi Y, Yajima Y, Kador PF, et al. Localization of aldose reductase in the human eye. Diabetes 1984;33:562-6.

9 Kaji Y, Usui T, Oshika T, et al. Advanced glycation end products in diabetic corneas. Invest Ophthalmol Vis Sci 2000;41:362-8.

10 Fujishima H, Shimazaki J, Yagi Y, et al. Improvement of corneal sensation and tear dynamics in diabetic patients by oral aldose reductase inhibitor, ONO2235: a preliminary study. Cornea 1996;15:368-72.

11 Fujishima H, Tsubota K. Improvement of corneal fluorescein staining in post cataract surgery of diabetic patients by an oral aldose reductase inhibitor, ONO-2235. Br J Ophthalmol 2002;86:860-3

12 Hosotani H, Ohashi Y, Yamada M, et al. Reversal of abnormal corneal epithelial cell morphologic characteristics and reduced corneal sensitivity in diabetic patients by aldose reductase inhibitor, CT-112. Am J Ophthalmol 1995; 119:288-94.

13 Yokoi N, Niiya A, Komuro A, et al. Effects of aldose reductase inhibitor CT112 on the corneal epithelial barrier of galactose-fed rats. Curr Eye Res 1997:595-9.

14 Miyata K, Amano S, Sawa M, et al. A novel grading method for superficial punctate keratopathy magnitude and its correlation with corneal epithelial permeability. Arch Ophthalmol 2003;121:1537-9.

15 Gekka M, Miyata K, Nagai Y, et al. Corneal epithelial barrier function in diabetic patients. Cornea 2004;23:35-7.

16 Yokoi N, Komuro A, Nishida K, et al. Effectiveness of hyaluronan on corneal epithelial barrier function in dry eye. $\mathrm{Br} J$ Ophthalmol 1997;81:533-6.

17 Yokoi N, Kinoshita S. Clinical evaluation of corneal epithelial barrier function with slit-lamp fluorophotometer. Cornea 1995; 14:485-9.

18 Yokoi N, Kinoshita S, Akiyama K. A new slit-lamp fluorophotometer for the clinical evaluation of corneal epithelial barrier function. Acta Soc Ophthalmol Jpn 1994;98:641-7.

19 Shimazaki J, Fujishima H, Yagi Y, et al. Effects of diclofenac eye drops on corneal epithelial structure and function after small-incision cataract surgery. Ophthalmology 1996;103:50-7

20 Shimazaki J, Shimmura S, Mochizuki K, et al. Morphology and barrier function of the corneal epithelium after penetrating keratoplasty: association with original diseases, tear function, and suture removal. Cornea 1999; 18:559-64

21 Göbbels M, Spitznas M, Oldendoerp J. Impairment of corneal epithelial barrier function in diabetics. Graefes Arch Clin Exp Ophthalmol 1989;227:142-4.

22 Alfonso EC, Rosa RH Jr. Fungal keratitis. In: Krachmer JH, Mannis MJ, Holland EJ, eds. Cornea. St Louis: Mosby, 1997:1253-65.

23 Hamada Y, Araki N, Koh N, et al. Rapid formation of advanced glycation end products by intermediate metabolites of glycolytic pathway and polyol pathway. Biochem Biophys Res Commun 1996;228:539-43.

24 Kawasaki Y, Fujii J, Miyazawa N, et al. Specific detections of the early process of the glycation reaction by fructose and glucose in diabetic rat lens. FEBS Lett 1998;441:116-20.

25 Suarez G, Rajaram R, Oronsky AL, et al. Nonenzymatic glycation of bovine serum albumin by fructose (fructation). Comparison with the Maillard reaction initiated by glucose. J Biol Chem 1989;264:3674-9. 